\title{
Spectroscopic study of a laser-produced lead plasma: experimental atomic transition probabilities for $\mathrm{Pb}$ III lines
}

\author{
C Colón \\ A Alonso-Medina \\ C Herrán-Martínez
}

\begin{abstract}
The optical emission from a laser produced plasma (LPP) generated by a $10640 \AA$ laser irradiation, with a flux of $2 \times 10^{10} \mathrm{~W} \mathrm{~cm}^{-2}$, on $\mathrm{Pb}$ targets was recorded and analysed between 2000 and $7000 \AA$; spectral lines emitted by the first two ionization species of Pb were studied in a wide range of plasma parameters. First experimental values of the atomic transition probabilities for ten spectral lines of $\mathrm{Pb}$ III have been determined by measurement of emission line intensities from an optically thin LPP of Pb in an atmosphere of Ar. The studied plasma has a temperature of about $25300 \mathrm{~K}$ and an electron density of $10^{17} \mathrm{~cm}^{-3}$. Local thermodynamic equilibrium conditions and plasma homogeneity has been checked.
\end{abstract}

\section{Introduction}

As is well known, data of transition probabilities are relevant not only for atomic structure research, but also for applications to astrophysics, plasma and laser physics, and also for analysis techniques. There has been continuous interest in the determination of transition probabilities and lifetimes of neutral and ionized Pb (Lotrian et al 1979, Gorshkov and Verolainem 1985, Alonso-Medina 1996, 1997). Oscillator strengths are computed for both the resonance transition $6 s^{2}{ }^{1} \mathrm{~S}_{0}-6 s 6 \mathrm{p}^{1} \mathrm{P}_{1}$ and the intercombination line $6 \mathrm{~s}^{2}{ }^{1} \mathrm{~S}_{0}-6 \mathrm{~s} 6 \mathrm{p}^{3} \mathrm{P}_{1}$ in the $\mathrm{Pb}$ III by Migdalek and Baylis (1985); Ansbacher et al 1988) measured lifetimes of the 6s6p ${ }^{1} \mathrm{P}_{1}$ and ${ }^{3} \mathrm{P}_{1}, 6 \mathrm{~s} 6{ }^{1} \mathrm{D}_{2}, 6 \mathrm{~s} 7 \mathrm{~s}^{1} \mathrm{~S}_{0}$ and $6 \mathrm{p}^{2}{ }^{1} \mathrm{D}_{2}$ levels of $\mathrm{Pb}$ III, and lifetimes of the $6 \mathrm{~s} 6{ }^{3} \mathrm{D}_{1,2,3}$ and $6 s 7 s^{3} \mathrm{~S}_{1}$ levels of $\mathrm{Pb}$ III have also been measured by Pinnington et al (1988), using beam-foil techniques.

In the case of lines corresponding to $6 \mathrm{~s} 7 \mathrm{p}$ levels of $\mathrm{Pb}$, data of relative transition probabilities are almost absent from the literature; this was the reason for this work, which presents the transition probabilities for ten lines corresponding to level $\mathrm{P}$ of $\mathrm{Pb}$ III from measurements of emission lines intensities in a plasma generated by focusing a laser beam on a sample of $\mathrm{Pb}$ in an atmosphere of $\mathrm{Ar}$. The results are compared with our theoretical values using the Coulomb approximation.

The interaction of a high-power laser beam with solid samples generates plasma on the surfaces of the targets with high temperatures and electron densities. From the experimental point of view, laser produced plasmas (LPP) have proved to be a valuable and versatile source 


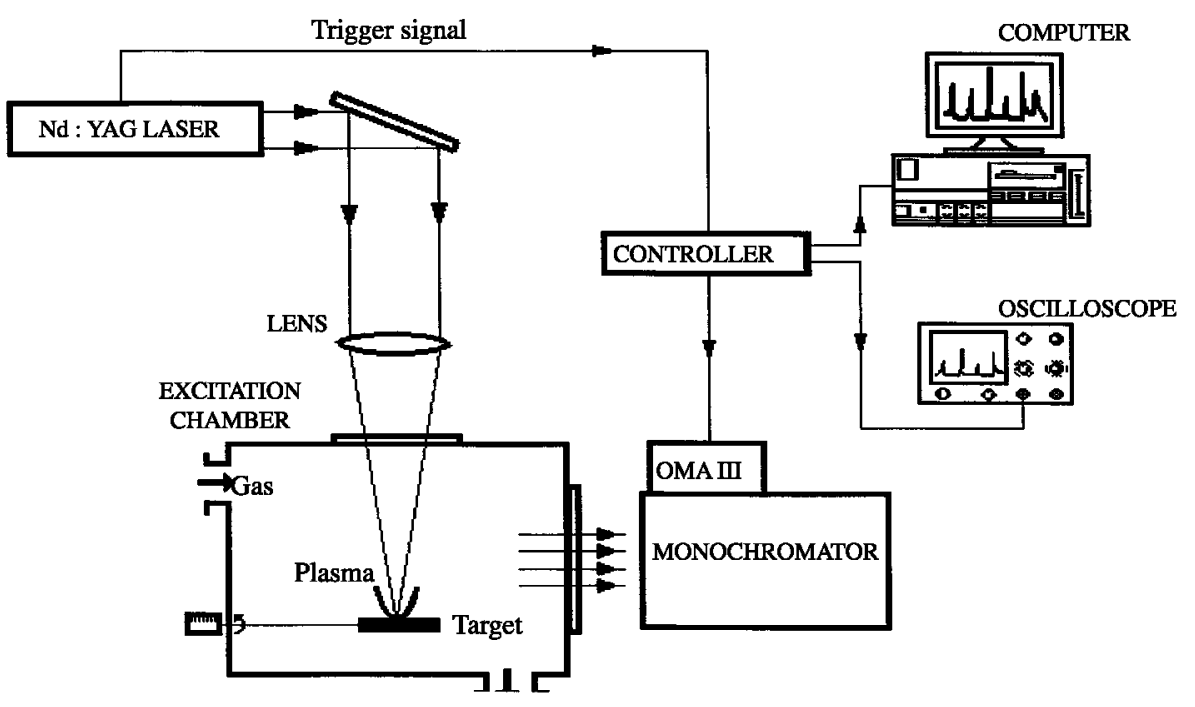

Figure 1. Schematic of the experimental set-up.

of spectroscopic data on ionized species-e.g. Radziemski and Cremers (1989), Blanco et al (1995), Alonso-Medina (1997), Ferrero et al (1997).

The homogeneity of this plasma is essential to obtain a uniform temperature and thus to interrelate the populations of various atomic energy levels, from which the transitions originate. The corresponding absolute transition probabilities were obtained from the line intensities of the $\mathrm{Pb}$ II spectrum under the LTE assumption. In this work the LTE assumption is discussed, and the homogeneity temperature and electron density of the plasma are studied. Plasma parameters such as temperature and electron density have been measured in different zones of the emitting region.

We will describe in section 2 the experimental system used for LPP spectroscopy, and in section 3 the various results obtained are discussed.

\section{Experimental set-up and experimental procedure}

The experimental system is similar as that described in previous papers (Alonso-Medina 1997, Blanco et al 1995, Ferrero et al 1997). A schematic diagram of the experimental arrangement is shown in figure 1 .

A lens of focal distance of $12 \mathrm{~cm}$ is used to focus, on a $\mathrm{Pb}$ target, the laser beam of a Nd:YAG laser, that generates $290 \mathrm{~mJ}$ pulses of $7 \mathrm{~ns}$ duration at frequency of $20 \mathrm{~Hz}$ and $10640 \AA$ wavelength. The laser irradiance on the blank was $2 \times 10^{10} \mathrm{~W} \mathrm{~cm}^{-2}$, the diameter of the standard crater was $0.5 \mathrm{~mm}$, and the spatial width of the focused laser beam was measured by recording its image with a 1024-element linear silicon diode array. The light emitted by the L.PP was transmitted through a sapphire window to the input slit of a $1 \mathrm{~m}$ Czemy-Turner spectrometer provided with a 2400 grooves $\mathrm{mm}^{-1}$ holographic grating. The resolution of the spectroscopic system was $0.13 \AA$ in the first order. The characteristics of the apparatus are shown in table 1.

Spectra were recorded by a time-resolved optical multichannel analyser (OMA III EG\&G) that allows the recording of spectra at a preset delay from the laser pulse and with a selected time length. Spectra were obtained at $0.15-0.80 \mu$ s delay from the laser pulse and light was 
Table 1. Experimental apparatus and characteristics.

\begin{tabular}{ll}
\hline Q-switched Nd:YAG Laser & Quantel YG 585 \\
Pulsewidth & $7 \mathrm{~ns}$ \\
Pulse energy & $290 \mathrm{~mJ}$ \\
Wavelength & $10640 \mathrm{~A}$ \\
Repetition rate & $20 \mathrm{~Hz}$ \\
& \\
Spectrometer & Spex 1704 \\
Type & $1 \mathrm{~m}$, Czerny-Turner \\
Grating & 2400 grooves mm ${ }^{-1}$ \\
Slit & $50 \mu \mathrm{m}$ \\
& \\
Detection system & EG\&G OMA III (model 1461) \\
Number of channels & 1024 \\
Minimum gate width & $100 \mathrm{~ns}$ \\
& \\
Fibre-optic cable & UV fused silica, incoherent bundle \\
Length & $5 \mathrm{~m}$ \\
Diameter & $1 \mathrm{~mm}$ \\
\hline
\end{tabular}

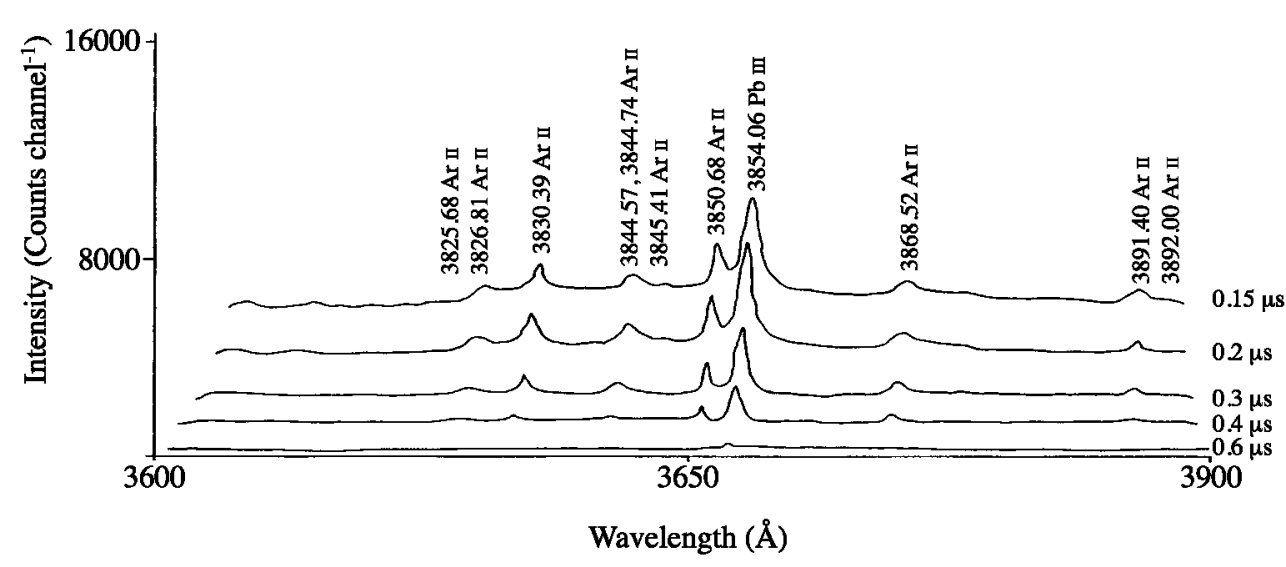

Figure 2. Section of typical emission spectrum from 0.15 to $0.6 \mu$ s delay time and 12 Torr atmosphere Ar.

collected during $0.1 \mu$ s in synchronism with the electronic trigger of the laser Q-switch. In each data acquisition period a correction was made with regard to the dark signal in the absence of the laser plasma. The instrumental profile of the line was determined with a precision of $97 \%$, being $0.11 \AA$ in second order; the instrumental width (full width at half maximum (FWHM)) for a wavelength of $3000 \AA$.

A chamber is used to generate the plasma, in a vacuum with a gas atmosphere; a vacuum of $10^{-5}$ Torr had also been attained inside the chamber by means of a turbomolecular pump, and it was filled with Ar and maintained at a constant pressure of 12 Torr throughout the measurement, using a small continuous flow of gas to maintain the purity of the atmosphere. The temperature, temporal evolution and electron density of LPP can be partially controlled by the use of suitable buffer gas (Alonso-Medina 1996, 1997, Zhao et al 1992, Wolf 1992), and the buffer gas pressure value, 12 Torr, was chosen because it gives the best contrast between line emission and continuum emission. Figure 2 presents a section of typical spectra. 


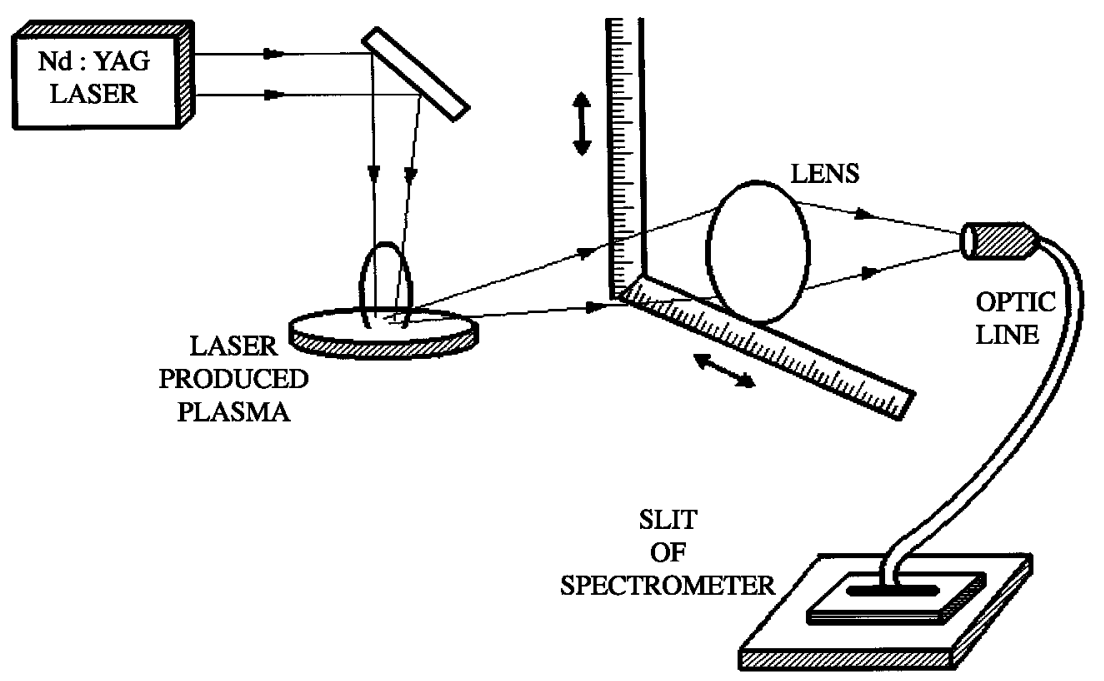

Figure 3. Schematics of the experimental arrangement for the study of plasma homogeneity.

The $\mathrm{Pb}$ sample, having a purity of $99.9 \%$, was located inside the chamber, on top of a device capable of moving it horizontally with respect to the laser beam, focused in such a way that the plasma was formed in each measurement on the smooth surface of the target and not on the centre formed during the previous measurement, figure 1.

The presence of traces of Ca, an impurity in the samples used, and moreover the use of $\mathrm{Ar}$ provides various spectrum lines with well known Stark parameters and transition probabilities, which will be useful to complete the determination of the temperature and electron density of plasma.

The same experimental system was used to study the homogeneity of the plasma but, in order to have spatial resolution, the light was focused by means of a lens on a $1 \mathrm{~mm}$ light guide being able to select the point of the plasma from which the light emission was observed. The measurements were taken by scanning the plasma emission in two perpendicular directions, as can be seen in figure 3 to determine where the different atomic species of $\mathrm{Pb}$ are located in the plasma and to determine the real values of the parameters of the plasma.

The spectral response of the system was obtained in the $2000-7000 \AA$ wavelength range by means of a previously calibrated lamp; deuterium was used for the 2000-4000 $\AA$ range and of tungsten for the 3500-7000 $\AA$ range. The stimulated error of this measurement was about $3 \%$.

The spectra were stored in a computer for further analysis, which was made by fitting the observed line shapes to numerically generated Voigt profiles. The instrumental profile was predetermined by observation of various narrow lines emitted by hollow cathode lamps. Local profiles were obtained after Abel inversion of the integrated intensity, Lochte-Holtgreven (1968). The fitting of the observed profiles provides the total intensity very accurately, as well as the broadening of the spectral lines.

In our experimental conditions - with a delay time of $0.4 \mu$ s after the laser pulse - in an atmosphere of $\mathrm{Ar}$ at 12 Torr all the transitions of the $\mathrm{Pb}$ III spectrum can be observed as those of the $\mathrm{Pb}$ I, $\mathrm{Pb}$ II, $\mathrm{Ar}$ пा, $\mathrm{Ar}$ II and the most intense transitions of the Ca Ir. Relative intensities have been measured evaluating the area under each one, for which purpose adjustments were made to the profiles observed of the lines by means of a convolution of the instrumental 


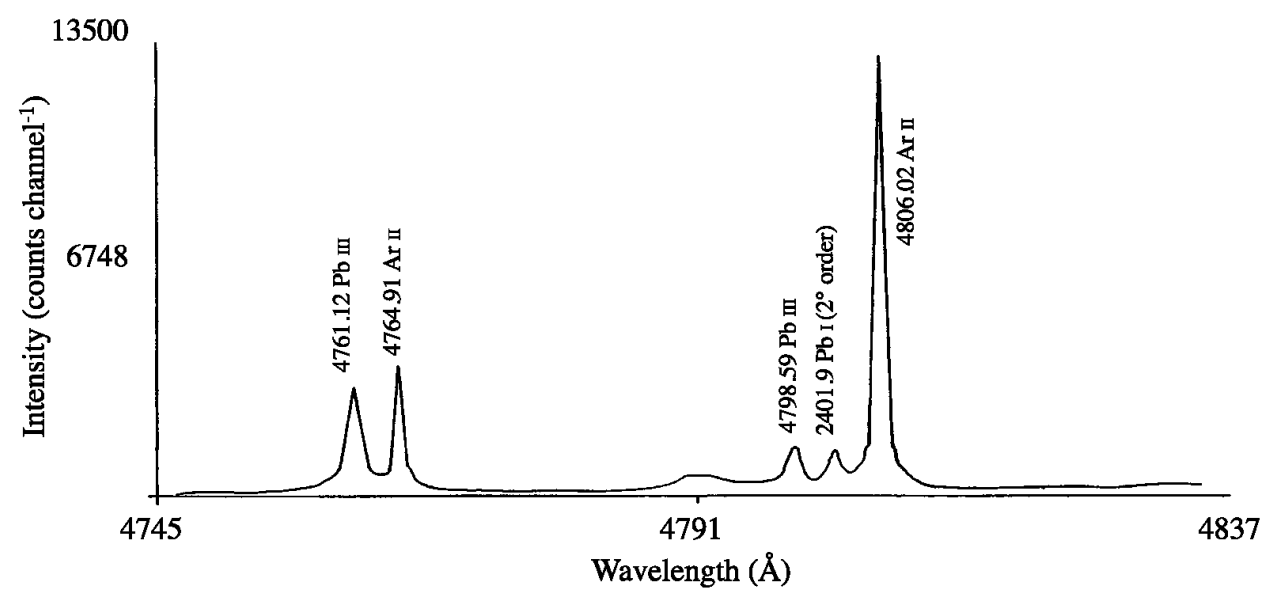

Figure 4. Typical $\mathrm{Pb}$ III spectra at 12 Torr $\mathrm{Ar}$ corresponding to $0.4 \mu$ s delay time.

profile, known, with the Voigt profile, obtained from the contributions selected, Lorentzian and Gaussian, Brüggemann and Bollig (1992).

Branching ratios have been determined measuring the relative emission line intensities for lines arising from the same upper levels $\left({ }^{2} \mathrm{~S}\right) 7 \mathrm{p}^{3} \mathrm{P}_{0,1,2}$ of $\mathrm{Pb}$ III. Figure 4 displays a typical emission spectrum obtained in the conditions described above.

\section{Experimental results and discussion}

When LTE conditions are verified the populations of the linked states follow Boltzmann distribution, which can be used as a first approximation to determine the temperature and the electron density, Griem (1974), Befeki (1976).

Assuming an optically thin layer and the presence of LTE the transition probabilities $A_{i, j}$ of a given species are related to the emitted line intensities by the expression:

$$
A_{i j}=\left[\frac{U(T)}{N g_{i}}\right] I_{i j} \mathrm{e}^{\frac{E_{i}}{k T}} ;
$$

$U(T)$ is the partition function of the species, $N$ is the number density of the species, $E_{i}$ is the excitation energy of upper level $i, g_{i}$ its statistical weight, $I_{i j}$ is the emitted intensity for the transition to lower $j$-in photons $\mathrm{s}^{-1}$ - and $k$ is the Boltzmann constant; the line intensities used are normalized to the highest value. Since in this experiment we want to determine only relative $A_{i j}$ values for lines of a given atomic species, we need only measure relative intensities, and to determine the value of $T$. If we plot $\ln \left(\frac{I_{i j}}{g_{i} A_{i j}}\right)$ versus $E_{i}$, Boltzmann plot, the resulting straight line would have a slope $-1 / k T$ and therefore the temperature can be obtained without having to know the total density of atoms or the partition function.

The energy of the different levels are those of Moore (1958). Figure 5 shows a partial energy level diagram for $\mathrm{Pb} \mathrm{III}$ and the figure 6 shows a partial energy level diagram for $\mathrm{Pb} \amalg$, showing transitions used for temperature calculation.

The designation and the $\mathrm{Pb}$ II, $\mathrm{Ar}$ II and $\mathrm{Ca}$ II transition probabilities selected for determination of the excitation temperature by Boltzmann plot are shown in table 2.

The plasma temperature has been determined by means of a Boltzmann plot for some lines of $\mathrm{Pb} \amalg$, the transitions probabilities of which were known (Alonso-Medina 1997), and was found to be $(24900 \pm 1600) \mathrm{K}$, figure 7 . For some lines of Ar II the transition probabilities of 


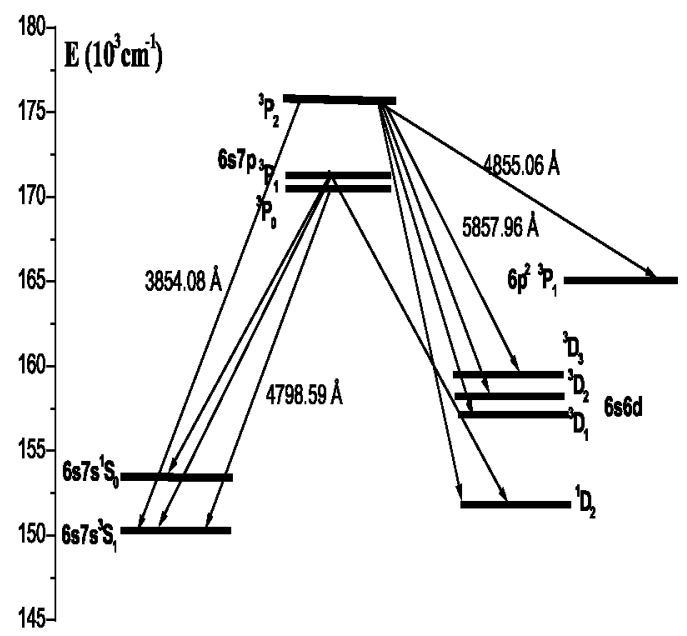

Figure 5. Partial energy-level diagram for $\mathrm{Pb}$ III showing the transitions in this study.

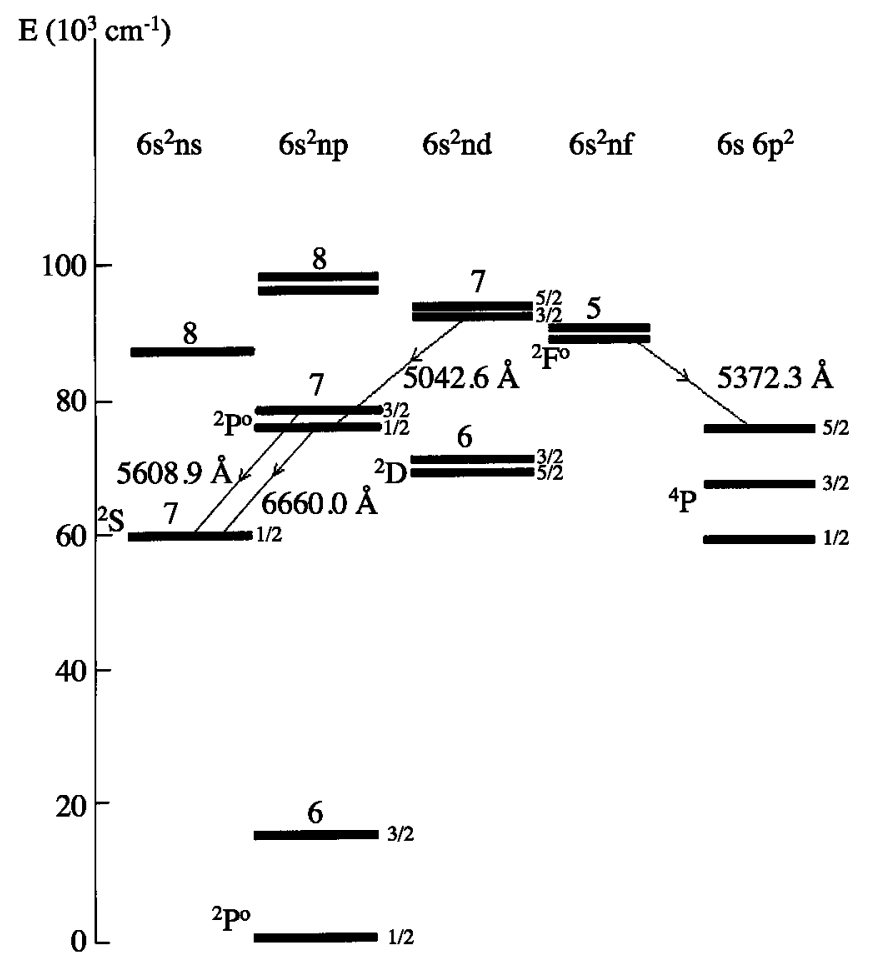

Figure 6. Partial energy-level diagram for $\mathrm{Pb}$ II showing transitions used for temperature calculations.

which were known (Vujnovic and Wiese 1992, Pellerin et al 1997), the plasma temperature was found to be ( $25300 \pm 1700) \mathrm{K}$; and with some lines of Ca II the transition probabilities of which were known (Wiese et al 1969), the plasma temperature was found to be (25500 \pm 2600$) \mathrm{K}$. These values are totally compatible. 
Table 2. Additional spectroscopic data of $\mathrm{Pb}$ II and $\mathrm{Ar}$ II employed for temperature determination.

\begin{tabular}{llcll}
\hline $\begin{array}{l}\text { Specie } \\
\text { atomic }\end{array}$ & $\begin{array}{l}\text { Wavelength } \\
(\AA)\end{array}$ & $\begin{array}{l}\text { Energy (eV) } \\
\text { (upper level) }\end{array}$ & $\begin{array}{l}g_{i} \\
\text { (upper level) }\end{array}$ & $\begin{array}{l}A_{i j} \\
\left(10^{6} \mathrm{~s}^{-1} \text { ) }\right.\end{array}$ \\
\hline Pb II & 6660.00 & 9.332 & 2 & $57.2 \pm 5.4^{\mathrm{a}}$ \\
& 5608.90 & 9.581 & 4 & $84.8 \pm 8.5^{\mathrm{a}}$ \\
& 5372.30 & 11.471 & 8 & $62.1 \pm 6.2^{\mathrm{a}}$ \\
& 5042.60 & 11.690 & 4 & $90.0 \pm 8.9^{\mathrm{a}}$ \\
Ar II & 4806.60 & 19.22 & 6 & $78.0 \pm 4 \%^{\mathrm{b}}$ \\
& & & & $78.2 \pm 5 \%^{\mathrm{c}}$ \\
& 4847.81 & 19.30 & 2 & $84.9 \pm 9 \%^{\mathrm{b}}$ \\
& & & & $88.9 \pm 5 \%^{\mathrm{c}}$ \\
& 3850.58 & 19.97 & 4 & $38.7 \pm 12 \%^{\mathrm{b}}$ \\
& & & & $43.6 \pm 5 \%^{\mathrm{c}}$ \\
& 4131.73 & 21.43 & 2 & $85.0 \pm 11 \%^{\mathrm{b}}$ \\
& & & & $96.7 \pm 15 \%^{\mathrm{c}}$ \\
& 3841.52 & 22.84 & 2 & $26.9 \pm 14 \%^{\mathrm{b}}$ \\
& & & & $24.5 \pm 10 \%^{\mathrm{c}}$ \\
Ca II & 3933.7 & 3.12 & 2 & $150 \pm 25 \%^{\mathrm{d}}$ \\
& 3736.9 & 6.46 & 4 & $165 \pm 25 \%^{\mathrm{d}}$ \\
& 5001.5 & 9.98 & 4 & $20 \pm 50 \%^{\mathrm{d}}$ \\
\hline
\end{tabular}

\footnotetext{
a Alonso-Medina (1997).

b Vujnovic and Wiese (1992).

${ }^{c}$ Pellerin et al (1997).

${ }^{\mathrm{d}}$ Wiese et al (1969).
}

The electron density, $N_{\mathrm{e}}$, of the plasma investigated has been obtained by comparing the Stark broadening for several transitions with those of other authors, using the expression by McWhirter (1963) and Sobelmann (1995):

$$
\Delta \lambda=2 \omega\left(\frac{N_{\mathrm{e}}}{10^{16}}\right)\left[1+1.75 A\left(\frac{N_{\mathrm{e}}}{10^{16}}\right)^{14}\left(1-1.2 N_{D}^{-13}\right)\right]
$$

where $\Delta \lambda$ is the FWHM of the transition considered, $\omega$ is the electron impact parameter, $A$ is the ion-broadening parameter. $N_{\mathrm{D}}$ is the number of particles in the Debye sphere and was estimated by $N_{\mathrm{D}}=1.38 \times 10^{3} T^{3 / 2} N_{\mathrm{e}}^{-1 / 2}$ which is well above the limit $N_{\mathrm{D}}=2$ of the Debye approximation for correlation effects, Wolf (1992), Sobelmann et al (1995).

The values of the electron density was obtained using in the last equation the experimental value of the electron impact parameter, $\omega$, for $4244.9 \AA \mathrm{Pb}$ II transition. This value of $\omega=1.50 \AA$ was obtained for a temperature of $24000 \mathrm{~K}$ and $N_{\mathrm{e}}=1.0 \times 10^{17} \mathrm{~cm}^{-3}$, (Salakhov et al 1984). The Stark broadening of this transition was obtained as the Lorentzian part, $\Delta \lambda=1.5 \AA$ of his Voigt profile. The electron density was $(1.01 \pm 0.19) \times 10^{17} \mathrm{~cm}^{-3}$.

The values of the electron densities from very different spectrum lines are in good agreement. The electron impact parameter for these lines was obtained by extrapolation in table 3 which displays the values obtained with the $5608.9,5544.3$ and $4244.9 \AA$ of $\mathrm{Pb}$ II, with 3928.6, 4764.9 and $4806.0 \AA$ of $\mathrm{Ar}$ II, and $3933.7 \AA$ of Ca II, with the third column, giving the Stark broadening $(\omega)$ obtained by Salakhov et al $(1984)$ and Puric et al $(1985,1990)$ for the lines of Pb II, obtained by Nick and Helbig (1986), Konjevic and Wiese (1990), Jalufka et al (1966) and Roberts (1968) for the lines of Ar II and the values obtained by Goldbach et al $(1983,1990)$ and Roberts and Eckerle (1967) for the line of Ca II. 


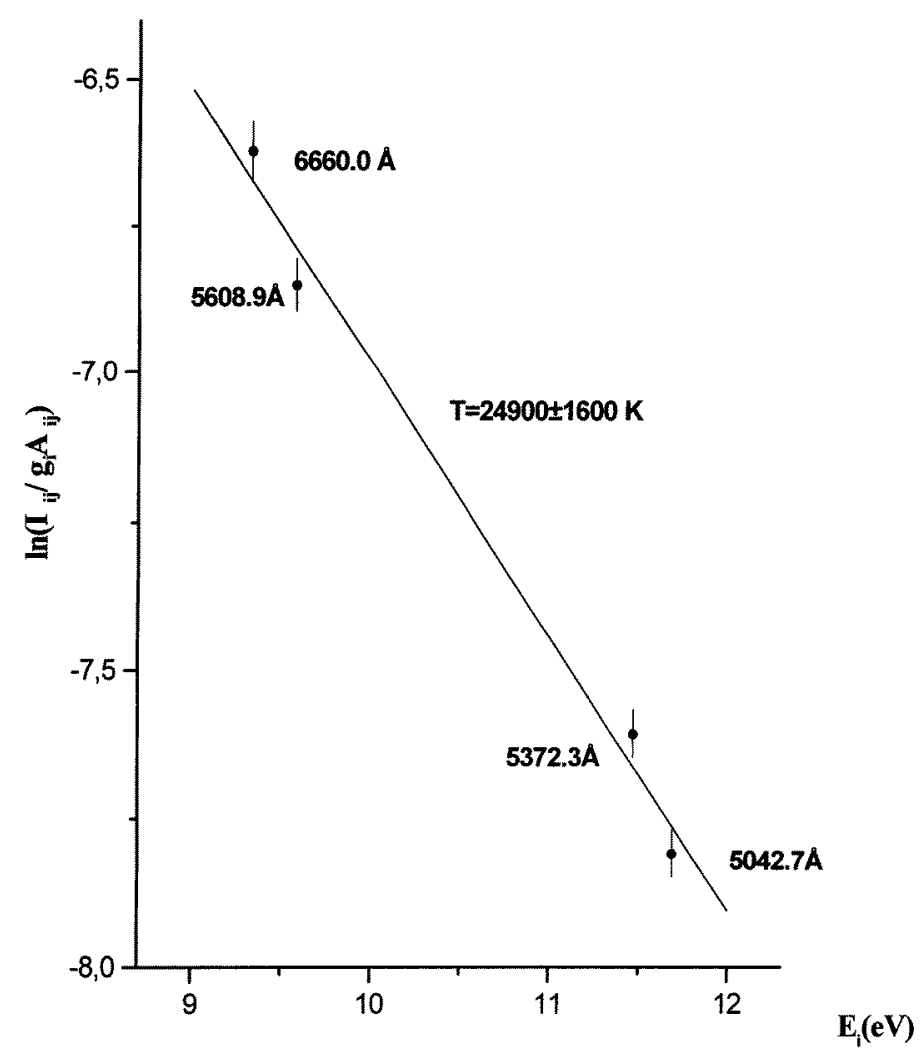

Figure 7. Boltzmann plot for $\mathrm{Pb}$ II lines emitted by a laser-produced plasma at $0.4 \mu$ s delay in $\mathrm{Ar}$ at a pressure of 12 Torr.

The electron densities obtained from the Stark broadening may be considered reliable because the other broadening mechanisms considered in this study barely account for $3 \%$ of the total broadening value.

The obtained value of $10^{17} \mathrm{~cm}^{-3}$ is sufficient to assume LTE for the population of the studied levels according to the criterion of McWhirter (1963):

$$
N_{\mathrm{e}}\left(\mathrm{cm}^{-3}\right) \geqslant 1.6 \times 10^{12} \sqrt{T}(\Delta E)^{3},
$$

where $\Delta E$, in $\mathrm{eV}$, is the energy difference between both configurations and $T$, in $\mathrm{K}$, the plasma temperature, and $N_{\mathrm{e}}$ the lower limit of the electron density necessary to maintain the populations of the energy level at 10\% of the LTE by collision, in competition with the radiative processes.

Using the values obtained for the lines of $\mathrm{Pb}$ II the critical $N_{\mathrm{e}}$ is $8.21 \times 10^{15} \mathrm{~cm}^{-3}$, using the values obtained of the line of $\mathrm{Ar}$ II the critical value is $1.21 \times 10^{16} \mathrm{~cm}^{-3}$ and using the value obtained for the line of Ca II is $8.10 \times 10^{16} \mathrm{~cm}^{-3}$.

As a confirmation, a result of $(25450 \pm 4500) \mathrm{K}$ was obtained by applying the Saha equation to the $\mathrm{Ar}$ II lines $5062.0 \AA, 5141.8 \AA$ and the $5191.8 \AA$ line of $\mathrm{Ar}$ III, using the transition probabilities given by Wiese et al (1969), and using a value of $1.2 \times 10^{17} \mathrm{~cm}^{-3}$ for the electron density.

As a further confirmation of the LTE hypothesis, in our experimental conditions with a delay time of $0.4 \mu$ s after the laser pulse, in an atmosphere of Ar at 12 Torr, we have measured 
Table 3. Electron density of plasma (12 Torr, delay time of $0.4 \mu \mathrm{s}$ ).

\begin{tabular}{lllll}
\hline $\mathrm{Pb}$ II transition & Wavelength $(\AA)$ & $\begin{array}{l}\text { Temperature } \\
\left(10^{3} \mathrm{~K}\right)\end{array}$ & $\begin{array}{l}N_{\mathrm{e}}=10^{17} \mathrm{~cm}^{-3} \\
\omega_{\text {exp }}(\AA) \pm 15 \%\end{array}$ & $\begin{array}{l}N_{\mathrm{e}}\left(10^{17} \mathrm{~cm}^{-3}\right) \\
24900 \mathrm{~K}\end{array}$ \\
\hline $5 \mathrm{f}^{2} \mathrm{~F}_{7 / 2}^{\mathrm{o}} \rightarrow 6 \mathrm{~d}^{2} \mathrm{D}_{5 / 2}$ & 4244.9 & 16 & $1.52^{\mathrm{a}}$ & $1.01 \pm 0.19$ \\
& & 20 & $1.28^{\mathrm{a}}$ & \\
$7 \mathrm{~d}^{2} \mathrm{D}_{5 / 2} \rightarrow 7 \mathrm{p}^{2} \mathrm{P}_{3 / 5}^{\mathrm{o}}$ & 5544.3 & 24 & $1.50^{\mathrm{b}}$ & \\
& & 16 & $3.18^{\mathrm{a}}$ & $1.10 \pm 0.11$ \\
$7 \mathrm{p}^{2} \mathrm{P}_{3 / 2}^{\mathrm{o}} \rightarrow 7 \mathrm{~s}^{2} \mathrm{~S}_{1 / 2}$ & 5608.9 & 20 & $2.10^{\mathrm{a}}$ & \\
& & 16 & $2.12^{\mathrm{a}}$ & $1.00 \pm 0.18$ \\
& & 20 & $1.72^{\mathrm{a}}$ & \\
$\mathrm{Ar}$ II transition & Wavelength $(\AA)$ & $\left(10^{3} \mathrm{~K}\right)$ & $\omega_{\mathrm{exp}}(\AA) \pm 30 \%$ & $\left(10^{17} \mathrm{~cm}^{-3}\right)$ \\
\hline${ }^{4}{ }^{4} \mathrm{~S}_{3 / 2} \rightarrow 4 \mathrm{~s}^{4} \mathrm{P}_{1 / 2}$ & 3928.62 & 12.8 & $0.164^{\mathrm{c}}$ & $1.30 \pm 0.37$ \\
$4 \mathrm{p}^{2} \mathrm{P}_{3 / 2}^{\mathrm{o}} \rightarrow 4 \mathrm{~s}^{2} \mathrm{P}_{1 / 2}$ & 4764.90 & 12.8 & $0.304^{\mathrm{c}}$ & $1.12 \pm 0.35$ \\
$4 \mathrm{p}^{4} \mathrm{P}_{5 / 2}^{\mathrm{o}} \rightarrow 4 \mathrm{~s}^{4} \mathrm{P}_{5 / 2}$ & 4806.00 & 12.8 & $0.238^{\mathrm{c}}$ & $1.15 \pm 0.36$ \\
& & 18 & $0.390^{\mathrm{d}}$ & \\
\hline & & 31 & $0.404^{\mathrm{e}}$ & \\
\hline $\mathrm{Ca}$ II transition & Wavelength $(\AA)$ & $\left(10^{3} \mathrm{~K}\right)$ & $\omega_{\mathrm{exp}}(\AA) \pm 30 \%$ & $\left(10^{17} \mathrm{~cm}^{-3}\right)$ \\
\hline $4 \mathrm{p}^{2} \mathrm{P}_{1 / 2}^{\mathrm{o}} \rightarrow 4 \mathrm{~s}^{2} \mathrm{~S}_{1 / 2}$ & 3933.7 & 12.8 & $0.10^{\mathrm{f}}$ & $1.26 \pm 0.36$ \\
& & 25 & $0.12^{\mathrm{g}}$ & \\
\hline
\end{tabular}

\footnotetext{
${ }^{a}$ Puric et al (1985).

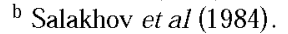

c Nick and Helbig (1986).

d Jalufka et al (1966)

e Roberts (1968).

${ }^{\mathrm{f}}$ Goldbach et al (1983).

$g$ Roberts and Eckerle (1967).
}

the plasma temperature of the studied alloy of $\mathrm{Pb}(0.5 \%)$ and $\mathrm{Sn}(95.5 \%)$, by applying the Saha equation to the $\mathrm{Pb}$ I line at $5005.5 \AA$ and the $5042.6 \AA$ and $5602.9 \AA$ lines of $\mathrm{Pb}$ II, using the transition probabilities given by Alonso-Medina $(1996,1997)$, and we have found a value of (25 $300 \pm 1500) \mathrm{K}$ for the temperature, and we have measured the electron density obtaining a value of $10^{17} \mathrm{~cm}^{-3}$. These values are totally compatible with the values obtained with the sample of $\mathrm{Pb}$ at $99.9 \%$ purity.

In order to determine the change of the temperature and the electron density in different regions of the plasma, several $\mathrm{Pb}$ II and $\mathrm{Ar}$ II lines were used. These results indicate a satisfactory homogeneity for temperature and electron density. Deviations from the average are less than $10 \%$ for $N_{\mathrm{e}}$ and less than $5 \%$ for $T$ in a region measuring approximately $2.5 \mathrm{~mm}$ in size corresponding to $95 \%$ of the emission of light; similar results have been obtained in other LPP experiments.

The determination of transition probabilities from the measurement of spectral line intensities requires the use of optically thin sources to avoid self-absorption of the plasma and can be made once the temperature and electron density are known by absorption coefficient calculations, Corney (1977). In this work self-absorption effects turned out to be lower than $3 \%$ for the most intense lines, and therefore the plasma can be considered as optically thin.

The transition probabilities obtained for ten lines of $\mathrm{Pb}$ III arising from some upper levels ( $\left.{ }^{2} \mathrm{~S}\right) 7 \mathrm{p}{ }^{3} \mathrm{P}_{0,1,2}$ with wavelengths in the range $3500-6000 \AA$ are displayed in column 3 of table 4 . 
Table 4. Experimental transition probabilities $\left(A_{i j}\right)$ of some lines of $\mathrm{Pb}$ III obtained by using the plasma temperature (12 Torr, delay time of $0.4 \mu \mathrm{s})$.

\begin{tabular}{cllcr}
\hline \multicolumn{2}{c}{ Transition } & & \multicolumn{2}{c}{$A_{i j}\left(10^{6} \mathrm{~s}^{-1}\right)$} \\
\cline { 5 - 6 } & & & Wavelength \\
Upper & Lower & $(\AA)$ & $\begin{array}{c}\text { This work (experimental) } \\
\text { (coulomb) }\end{array}$ \\
\hline $7 \mathrm{p}^{3} \mathrm{P}_{0}^{0} \rightarrow$ & $7 \mathrm{~s}^{3} \mathrm{~S}_{1}$ & 4798.59 & $172.1 \pm 17.2$ & 132.0 \\
$7 \mathrm{p}^{3} \mathrm{P}_{1}^{\mathrm{o}} \rightarrow$ & $7 \mathrm{~s}^{3} \mathrm{~S}_{1}$ & 4761.12 & $118.8 \pm 11.9$ & 134.9 \\
& $6 \mathrm{~d}^{1} \mathrm{D}_{2}$ & 5207.10 & $35.6 \pm 4.0$ & \\
& $7 \mathrm{~s}^{1} \mathrm{~S}_{0}$ & 5779.41 & $117.9 \pm 11.8$ & \\
$7 \mathrm{p}{ }^{3} \mathrm{P}_{2}^{0} \rightarrow$ & $7 \mathrm{~s}^{3} \mathrm{~S}_{1}$ & 3854.08 & $210.9 \pm 21.1$ & 234.9 \\
& $6 \mathrm{~d}^{1} \mathrm{D}_{2}$ & 4141.59 & $14.9 \pm 1.6$ & \\
& $6 \mathrm{p}^{2} \mathrm{P}_{1}$ & 4855.06 & $9.9 \pm 1.3$ & 27.4 \\
& $6 \mathrm{~d}^{3} \mathrm{D}_{1}$ & 5380.12 & $2.2 \pm 0.4$ & 0.8 \\
& $6 \mathrm{~d}^{3} \mathrm{D}_{2}$ & 5523.97 & $63.4 \pm 6.8$ & 11.5 \\
& $6 \mathrm{~d}^{3} \mathrm{D}_{3}$ & 5857.96 & $65.5 \pm 6.9$ & 57.7 \\
\hline
\end{tabular}

Absolute transition probabilities were obtained from relative line strength measurements by comparison with the relative line strength of the $5608.9 \AA \mathrm{Pb}$ пा transition in the Saha equation. Quoted errors in this table have been calculated as the quadratic addition of the statistical uncertainties the error due to the temperature estimate and systematic errors. We included our calculations obtained using the Coulomb approximation for comparison. These estimates were in relative agreement with the present experiment. It would be interesting if more rigorous calculations could explain the particular discrepancies.

\section{Acknowledgments}

It is a pleasure to acknowledge stimulating discussions with Professor J Campos; this work was supported by the Departamento de Fisica Atómica, Molecular y Nuclear (Facultad de CC Fisicas, UCM, Spain). 